Ergebnisse der Enquete-Kommission „Schutz des Menschen und der Umwelt"

\section{Konzept Nachhaltigkeit}

\section{Nach mehr als drei Jahren Arbeit, Diskussionen, Anhörungen von Expertlnnen und diversen Studien hat die Enquete-Kommission "Schutz des Menschen und der Umwelt" Ende Juni ihren Abschlußbericht präsentiert.}

$\mathrm{D}$

Von Sabine Lebmann

ie Aufgaben der Kommission lagen u.a. in der Formulierung von Umweltqualitätsund Umwelthandlungszielen, der Erarbeitung von Grundlagen für einen nationalen Umweltplan, sowie in der Identifierung von Handlungsspielräumen und Innovationspotentialen.

Die Enquete-Kommission betont in ihrem Bericht (1) die Dreidimensionalität der nachhaltig zukunftsfähigen Entwicklung und schlägt als institutionelle Reform einen Rat für Nachhaltigkeit mit Anbindung an das Bundeskanzleramt vor. Auf diese Weise soll das Thema zur Querschnittsaufgabe der Politik und die ökologische, soziale und ökonomische Dimension der Nachhaltigkeit zukünftig stärker integriert werden. Vor dem Hintergrund dieser drei Dimensionen hat die Enquete-Kommission nun auch soziale und ökonomische Regeln vorgeschlagen. So wird z.B. gefordert, daß das ökonomische System individuelle und gesellschaftliche Bedürfnisse effizient befriedigen soll, Preise die Knappheit der Ressourcen und der Produkte wiedergeben und die ökonomische Leistungsfähigkeit der Gesellschaft erhalten werden sollen (2). Aus sozialer Sicht werden die Bedingungen für die Leistungsfähigkeit einer solidarischen Gesellschaft formuliert. Die vier bereits bestehenden ökologischen Regeln zum Umgang mit Stoffen wurden um eine fünfte Regel „Gefahren und unvertretbare Risiken für die menschliche Gesundheit durch anthropogene Einwirkungen sind zu vermeiden" ergänzt.

Hinsichtlich der Grundlagen für einen nationalen Umweltplan hat die Kommission konkrete Zielvorschläge nur zur Reduzierung der Versauerung von Böden und zum Flächenverbrauch gemacht. Der Zuwachs des Flächenverbrauchs soll bis zum Jahre 2010 auf 10 Prozent (bezogen auf die Rate von 1993 bis 1995) reduziert werden und etwa 15 Prozent der Gesamtfläche der Bundesrepublik sollen als Naturschutz- und Freifläche reserviert werden. Zur Verminderung der weiteren Zersiedelung fordert die Kommission die Stärkung der städtischen Strukturen.
Wohnen und Arbeiten sind räumlich wieder enger zu verbinden, Baulücken zu schließen und die Wiedernutzung von Industrie- und Gewerbebrachen durch Sanierung zu fördern. Ebenfalls soll der Baubestand verstärkt gefördert werden. Dies hat gleichzeitig positive Beschäftigungseffekte, da die Bestandserneuerung im Altbau mehr Arbeitsplätze schafft als der Neubau. Weiter soll die Förderung des Wohnungsbaus stärker an ökologische Kriterien des Flächen-, Ressourcen- und Energieverbrauchs gebunden werden. Auf eine Empfehlung der Energiebesteuerung zur Reduzierung des Energieverbrauchs und der Kohlendioxidemissionen im Gebäudebereich konnten sich die Kommissionsmitglieder nicht einigen.

Als Strategie gegen die Versauerung von Böden empfiehlt die Kommission, einen Vorschlag der Kommission der Europäischen Gemeinschaften aufzugreifen. Danach sind bis zum Jahre 2010 die Emissionen von Stickoxiden um 59 Prozent, Schwefeldioxid um 92 Prozent und Ammoniak um 58 Prozent $\mathrm{zu}$ reduzieren. Allerdings wollte die Mehrheit der Kommission den Vorschlag nicht direkt zu übernehmen, sondern nur als Diskussionsgrundlage für ein Umwelthandlungsziel formulieren. Die Mehrheit der Kommission konnte sich auch nicht auf konkrete Maßnahmenvorschläge für die Landwirtschaft einigen und fällt damit sogar hinter die Vorschläge von Umweltministerin Angela Merkel zurück. SPD und Bündnis 90/Die Grünen forderten deshalb in einem Sondervotum die stärkere Förderung des ökologischen Landbaus und die Einführung einer Stickstoffabgabe. Zur Reduzierung der Emissionen im Verkehr wird eine Erhöhung der Mineralölsteuer sowie ein Tempolimit empfohlen.

Maßnahmen wurden auch für den Bereich der Informations- und Kommunikationstechnik vorgeschlagen. Hier ist die konsequente Ausgestaltung und Umsetzung der Produktverantwortung im Sinne einer vollständigen Rücknahmeverpflichtung gefordert. In diese sollten zukünftig auch Kleingeräte, Elektro-Haushalt-Großgeräte und Unterhaltungselektronik einbezogen wer- den. Produktkennzeichnung, Gerätepaß und Verbraucherinformation können den Kauf von umweltverträglichen Geräten und deren Nutzung anregen. Wichtig sind im Bereich der I+K-Technik auch die Ermöglichung des breiten Zugangs zu Informationen und die BenutzerInnenfreundlichkeit, um eine Spaltung der Gesellschaft in „Informationsarme“ und „Informationsreiche“ zu vermeiden und die Chance für eine verbesserte Politikbeteiligung zu gewährleisten.

Die Enquete-Kommission hat mit ihrem Bericht einige gute Ansätze für den Weg zu einer nachhaltig zukunftsfähigen Entwicklung geliefert. Die vorgeschlagenen Ziele und Maßnahmen sind aber nicht ausreichend und bleiben zum Teil sogar hinter nationalen und internationalen Diskussionen zurück. So konnte sich die Kommission nicht auf die Formulierung von Ansätzen für eine Energieund Mineralölbesteuerung einigen. Die ökonomischen und sozialen Regeln zur Nachhaltigkeit enthalten keine Ansätze für die erforderlichen Veränderungen von Wirtschafts- und Konsumweisen. Bei der Betonung der Gleichrangigkeit der drei Dimensionen wird die Gefahr vernachlässigt, daß der Umweltschutz weiterhin kurzfristigen wirtschaftlichen Interessen nachgestellt wird, anstatt eine tatsächliche Integration zu erreichen. Diese Kritik hat Jürgen Rochlitz (Bündnis 90/ Die Grünen) in einem Sondervotum festgehalten. Der Entwicklungsaspekt der Nachhaltigkeit fehlt im Bericht völlig. Trotz dieser Defizite läßt das erfahrungsgemäß starke Interesse an den Berichten der Enquete-Kommissionen hoffen, daß auch der vorliegende Bericht einen wichtigen Beitrag zur weiteren Nachhaltigkeitsdiskussion liefert.

\section{Anmerkungen}

(1) Enquete-Kommission "Schutz des Menschen und der Umwelt - Ziele und Rahmenbedingungen einer nachhaltig zukunftsverträglichen Entwicklung": Konzept Nachhaltigkeit - Vom Leitbild zur Umsetzung. Abschlubbericht. BT-Drucksache $13 / 11200$.

(2) Mit den Thema der ökonomischen Dimension der Nachhaltigkeit wird sich der Schwerpunkt der nächsten Ausgabe 6/98 von Ökologisches Wirtschaften näher beschäftigen.

\section{Die Autorin}

Sabine Lehmann ist Mitarbeiterin der Bundestags-

fraktion Bündnis $90 /$ Die Grünen.

Kontakt: Sabine Lehmann, Bündnis 90/Die Grünen, Bundestagsfraktion, Bundeshous Rheinweg 6 , 53113 Bonn, Tel. 02 28/1 648974, Fax. $0228 / 1646216$

E-Mail: sabine.lehmann@gruenebt.de 
(c) 20I0 Authors; licensee IÖW and oekom verlag. This is an article distributed under the terms of the Creative Commons Attribution Non-Commercial No Derivates License (http://creativecommons.org/licenses/by-nc-nd/3.o/), which permits unrestricted use, distribution, and reproduction in any medium, provided the original work is properly cited. 